\section{ORIGINAL RESEARCH}

J.H. Park

H.-S. Kang

M.H. Han

P. Jeon

D.-S. Yoo

T.H. Lee,

for the Korean

HydroSoft Registry

Investigators

\title{
Embolization of Intracranial Aneurysms with HydroSoft Coils: Results of the Korean Multicenter Study
}

BACKGROUND AND PURPOSE: Various modifications of detachable coils have been attempted to reduce recurrence rates in aneurysmal coil embolization and HydroSoft coil is one of them. The authors report their experience using HydroSoft coils in the treatment of cerebral aneurysms.

MATERIALS AND METHODS: The present study is a prospective multicenter observational series of 127 aneurysms in 120 patients treated with HydroSoft coils.

RESULTS: Ten centers participated in this study, and there were 92 (72\%) unruptured and 35 ruptured aneurysms. Aneurysm volumes were $\geq 100 \mathrm{~mm}^{3}$ in 21 (17\%) and $<100 \mathrm{~mm}^{3}$ in 106 (83\%) (mean, 56 $\mathrm{mm}^{3}$; range, $5-249 \mathrm{~mm}^{3}$ ). The average percentage length of HydroSoft coils detached in treated aneurysms was 67\% (range, 42\%-100\%). Immediate postprocedural angiography demonstrated complete aneurysm occlusion in 69\% (87/127 aneurysms), residual neck in 20\% (25/127), and residual sac in 12\% (15/127). Procedure-related adverse events occurred in 4.7\% (6/127 aneurysms), including procedural bleeding (5/127) and thromboembolism (2/127), and immediate procedure-related morbidity and mortality rates were $0.8 \%$ and $0 \%$. Conventional angiography or MRA follow-up was performed in $83 \%(105 / 127)$ at $\geq 6$ months after treatment (mean interval, 11 months; range, 6-24 months). The overall recanalization rate was 3\% (3/105 aneurysms; 2 major and 1 minor recanalizations). Progression to complete aneurysmal occlusion was noted in 20 of 27 aneurysms (74\%) during the follow-up.

CONCLUSIONS: The safety profile of HydroSoft coils appears acceptable. In terms of initial occlusion rates and durability, embolization by using HydroSoft coils seems to be favorable compared with most large series of pure platinum or coated coils.

ABBREVIATIONS: ISAT = International Subarachnoid Aneurysm Trial; MRA = MR angiography; PGA = polyglycolic; PGLA = combined polyglycolic/polylactic acid

S ince the introduction of Guglielmi detachable coils in 1991 (Boston Scientific, Natick, Massachusetts), endovascular treatment with detachable coils has been established as the mainstay of therapeutic options in cerebral aneurysms. The results of the International Subarachnoid Aneurysm Trial $(\text { ISAT })^{1}$ stressed coil embolization as a main treatment option for the management of ruptured intracranial aneurysms. However, long-term durability remains as a major limitation of this less invasive alternative to aneurysm clipping. Recurrence rates of $11 \%-36 \%$ have been reported with various coils. $^{2-5}$ Low packing attenuation of coils at the end of the initial procedure is proposed to be one of the main reasons for recurrence. To overcome this limitation, a number of techniques and devices have been tried, including intravascular stent placement ${ }^{6,7}$; a liquid embolic agent, Onyx (ev3, Irvine, California), reconstruction ${ }^{8}$; and balloon-remodeling tech-

Received November 10, 2010; accepted after revision February 7, 2011.

From the Department of Neurosurgery (J.H.P.), Kangwon National University College of Medicine, Chuncheon, Korea; Departments of Neurosurgery (H.-S.K.) and Radiology (M.H.H.), Seoul National University College of Medicine, Seoul, Korea; Department of Radiology (P.J.), Sungkyunkwan University School of Medicine, Seoul, Korea; Department of Neurosurgery (D.-S.Y.), The Catholic University of Korea College of Medicine, Uijeongbu, Korea; and Department of Diagnostic Radiology (Korean HydroSoft Registry Investigators), School of Medicine, Pusan National University, Pusan, Korea.

This work was funded by MicroVention, Tustin, California, and was supported by the Korean Society of Interventional Neuroradiology.

Please address correspondence to Moon Hee Han, MD, PhD, Department of Radiology, Seoul National University College of Medicine, 28 Yeongeon-dong, Jongno-gu, Seoul 110-744, Korea; e-mail: hanmh@snuh.org

http://dx.doi.org/10.3174/ajnr.A2633 niques. ${ }^{9}$ Also, in an attempt to reduce recurrence rates, various modified coils have been developed and used, including those containing PGA or PGLA and a hydrogel-coated coil.

To improve coil packing attenuation, a new design of hydrogel-coated coils has become commercially available. The HydroSoft coil (Microvention Terumo, Tustin, California) is constructed as a platinum coil with an inner core of hydrogel and a stretch-resistant filament (Fig 1). This new coil is different from the HydroCoil (Microvention Terumo) in that it is softer, contains less hydrogel, and swells more slowly. Due to these properties, reposition and retrieval of the HydroSoft coils does not have time restrictions. The present study was designed to investigate the safety and efficacy of the HydroSoft coils as well as to evaluate the follow-up results.

\section{Materials and Methods}

\section{Study Design}

This proposed registry, conducted by the Korean Society of Interventional Neuroradiology, is a prospective multicenter registry of patients harboring intracranial aneurysms measuring $<10 \mathrm{~mm}$ in diameter. Approval for this prospective multicenter observational study was granted by the institutional review board. Patients treated with HydroSoft coils for intracranial aneurysms were registered to an independent central core laboratory during the period from July 2008 to July 2009. The primary end points were procedure-related adverse events and major aneurysmal recanalization on follow-up angiography (conventional or MRA) 6 months and 1 year after treatment. The inclusion criteria were as follows: 1) ruptured or unruptured intra- 

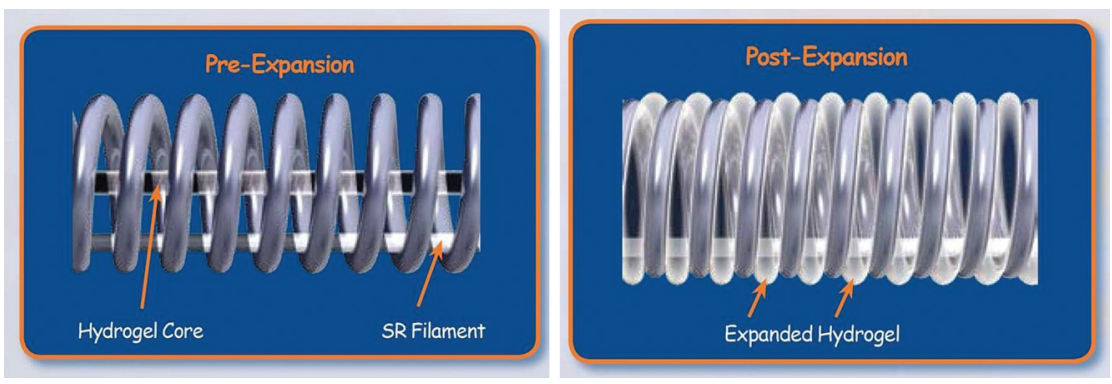

Fig 1. Pre- and postexpansion state of the HydroSoft coil. This platinum coil consists of an inner core of hydrogel and a stretch-resistant filament. It expands and fills up to $40 \%$ more volume than a bare platinum coil.

\begin{tabular}{lc}
\hline \multicolumn{2}{l}{ Table 1: Demographics of patients treated with HydroSoft coils } \\
\hline No. of Patients & 120 \\
\hline Sex (M:F) & $43: 77$ \\
Age, yr & $56.6 ; 30-80$ \\
$\quad$ mean; range & 127 \\
No. of treated aneurysms & $35(28 \%)$ \\
$\quad$ Ruptured & $92(72 \%)$ \\
Unruptured & \\
Aneurysm location & $69(54 \%)$ \\
Internal carotid artery & $41(33 \%)$ \\
Anterior cerebral artery & $8(6 \%)$ \\
Middle cerebral artery & $9(7 \%)$ \\
Posterior circulation & \\
Aneurysm size & $64(50 \%)$ \\
$<5$ mm & $54(43 \%)$ \\
5 mm $<8$ mm & $9(7 \%)$ \\
8 mm $<10$ mm & \\
Aneurysm volume & $55 \mathrm{~mm}^{3} ; 5-249$ \\
mean; range & $106(83 \%)$ \\
$<100 \mathrm{~mm}^{3}$ & $21(17 \%)$ \\
\hline$\geq 100 \mathrm{~mm}^{3}$ &
\end{tabular}

cranial aneurysm, 2) maximal aneurysmal diameter of $\leq 10 \mathrm{~mm}$, 3) percentage length of the HydroSoft coils detached (ie, the length of the HydroSoft coils inserted into an aneurysm divided by the total length of coils) of $\geq 40 \%$ in aneurysms of $8-10 \mathrm{~mm}$ and $<5 \mathrm{~mm}$ in diameter and $50 \%$ in aneurysms of $>5-8 \mathrm{~mm}$ in diameter, and 4) Hunt and Hess grades of $0-3$. The exclusion criteria were as follows: 1) embolization with any other coated coils; 2) staged embolization; 3) repeat embolization for recanalized or recurrent aneurysms; 4) thrombosed aneurysms; 5) fusiform, infectious, traumatic, or dissecting aneurysms; and 6) patients younger than 18 years of age.

The differences in percentage length were designed to accommodate framing coils in smaller aneurysms and to allow larger framing and filling coils in the larger aneurysms because the largest diameter of the HydroSoft coil is $6 \mathrm{~mm}$.

A reviewer (J.H.P) at the independent core laboratory scrutinized the data regarding the registered cases and decided whether the patients met the inclusion criteria and not the exclusion criteria. Clinical and radiologic follow-up data submitted to the core laboratory were critically reviewed.

\section{Clinical and Angiographic Features}

Patients' characteristics, including sex, age, and mode of presentation, were documented along with the Hunt and Hess grade. The aneurysm location, maximal diameter, volume, and the lengths of both HydroSoft and bare platinum coils were documented.

\begin{tabular}{lccc}
\hline \multicolumn{4}{l}{ Table 2: Procedure-related complications in $\mathbf{1 2 7}$ procedures } \\
\hline Complications & No. & Morbidity & Mortality \\
\hline Thromboembolism & 2 & 1 & 0 \\
Procedural bleeding & $5^{*}$ & 0 & 0 \\
Total & $6(4.7 \%)$ & $1(0.8 \%)$ & $0(0 \%)$ \\
\hline
\end{tabular}

* One patient showed procedural bleeding as well as thromboembolism.

Table 3: Degree of aneurysmal occlusion at immediately postprocedure and at the last follow-up

\begin{tabular}{lcccc}
\hline \multirow{2}{*}{$\begin{array}{l}\text { Rngiographic } \\
\text { Results }\end{array}$} & Immediate & Stationary & Improved & Worse \\
\cline { 3 - 5 } Class 1; complete & $87(69 \%)$ & $72(97)$ & NA & $2(3)$ \\
Class 2; residual neck & $25(19 \%)$ & $5(28)$ & $12(67)$ & $1(5)$ \\
Class 3; residual sac & $15(12 \%)$ & $4(31)$ & $9(69)$ & NA \\
Total & 127 & $81(79)$ & $21(18)$ & $3(3)$ \\
\hline
\end{tabular}

Note:-The numbers in parentheses are percentages.

\section{Endovascular Treatment}

Endovascular coil treatment was performed following a standardized protocol in the neuroangiography suite, as described previously. ${ }^{10,11}$ In most cases, general anesthesia was induced. In unruptured cases, systemic anticoagulation with heparin infusion was used from the beginning of the embolization. In ruptured cases, however, heparin infusion was started only after protective coil packing had been accomplished. Heparin was administered intravenously just after introduction of the sheath, first as a 3000-U bolus followed by $1000 \mathrm{U} / \mathrm{h}$. Heparin was discontinued after the procedure.

HydroSoft coil size and length selection were based on operator preference. Generally, bare platinum coils were used for framing, but in small-sized aneurysms, HydroSoft coils were readily deployed for the initial coil basket. HydroSoft coils were preferentially deployed until the coils defied deployment into an aneurysm or undue friction was felt.

\section{Angiographic Evaluation}

Immediate and follow-up angiographic results were recorded. The authors used the classification system by Roy et $\mathrm{al}^{12}$ : class 1 , complete occlusion; class 2, aneurysm neck opacification; and class 3 , aneurysm sac opacification. For each aneurysm, the pre- and postembolization angiograms were independently reviewed at an independent core lab for determination of aneurysm occlusion grades.

\section{Follow-Up Protocol}

Control angiograms were acquired immediately after treatment and were used as the baseline for comparison with follow-up studies. For all the patients, plain radiography was requested at 3 months after 

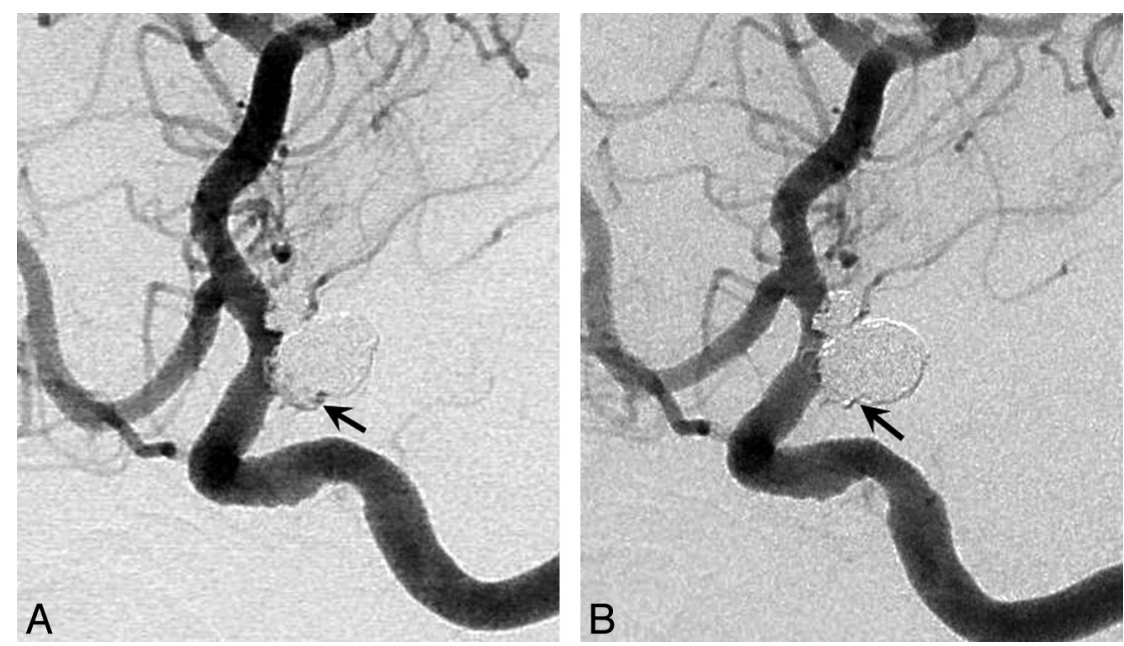

Fig 2. Improved degree of occlusion. A, A 70-year-old woman with elective coiling of a posterior communicating artery aneurysm. Postembolization angiogram shows residual filling into the aneurysm sac (arrow). B, A 7-month follow-up angiogram shows complete obliteration of the aneurysm lumen (arrow).

treatment with the same projections used at the time of coil embolization. Changes in the configuration of the coil mass prompted the investigators to request an immediate study such as MRA or conventional angiography. Otherwise, conventional angiography, preferably, or MRA was performed at $\geq 6$ months after treatment. 3D timeof-flight MRA was performed as a standard technique. "Major recanalization" was defined as contrast filling within the aneurysm dome or significant coil compaction, and "minor recanalization" was defined as minimal coil compaction at the aneurysmal neck.

\section{Statistical Analysis}

Simple and multiple logistic regression analyses were performed to find factors related to aneurysmal recanalization by using the Statistical Package for the Social Sciences (Version 12.0 for Windows; SPSS, Chicago, Illinois). A $P$ value $<.05$ was considered significant.

\section{Results}

In this prospective registry, 120 patients harboring 127 intracranial aneurysms were treated with the HydroSoft coils in 10 centers. Table 1 summarizes the characteristics of patients and aneurysms. There were 77 female patients (64\%) among 120, and the mean age was 56.6 years (range, $30-80$ years). There were $92(72 \%)$ unruptured and 35 ruptured aneurysms. Aneurysm volumes were $<100 \mathrm{~mm}^{3}$ in 106 (83\%) (mean, 55 $\mathrm{mm}^{3}$; range, $5-249 \mathrm{~mm}^{3}$ ).

\section{Procedure-Related Events}

Table 2 summarizes procedural complications. Procedure-related adverse events occurred in 4.7\% (6/127 aneurysms), including procedural bleeding (5/127) and thromboembolism (2/127); and the immediate procedure-related morbidity and mortality rates were $0.8 \%$ and $0 \%$, respectively.

Thromboembolism occurred in 2 procedures. In 1 case, the resultant cerebral infarction was asymptomatic. The other case had thrombosis at the distal basilar artery during treatment of a ruptured basilar artery aneurysm, and thrombolysis resulted in rebleeding from a coiled aneurysm. Although he had a temporary neurologic deficit, the patient was fully recovered at discharge. Otherwise, aneurysm perforation or procedural leakage was handled with immediate reversal of anticoagulation and complete coil packing, without leaving new neurologic deficits.

\section{Treatment and Immediate Angiographic Outcomes}

The overall mean percentage length of the HydroSoft coils was $67 \%$ (range, $42 \%-100 \%$ ). The mean packing attenuation was $42 \%$ (range, $19 \%-111 \%$ ). The degree of aneurysmal occlusion at the end of coil embolization was class 1 in $69 \%$ (87 of 127 aneurysms), class 2 in 19\% (25/127), and class 3 in 12\% (15/ 127) (Table 3).

\section{Radiologic Follow-up Results}

Radiologic follow-up data performed $\geq 6$ months after coil embolization were available for 105 aneurysms (83\%) ( $\geq 12$ months for 46 aneurysms and 24 months for 10 aneurysms; mean interval, 11 months; 24 aneurysms with conventional angiography and 81 with MRA) and revealed stable occlusion in $92(97 \%)$ aneurysms, minor recanalization in $1(1 \%)$, and major recanalization in 2 (2\%) (Table 3$)$. Comparison of occlusion classes immediately after embolization and at the last follow-up demonstrated stationary or improved class (Figs 2 and 3 ) in $98 \%$ of the aneurysms and a worse class (Fig 4) in $2 \%$ (Table 3). Of the 27 class 2 and 3 aneurysms, 20 (74\%) were improved to class 1 . Recanalized aneurysm profiles are summarized in Table 4. The logistic regression analysis revealed that ruptured aneurysmal status was the only significant factor related to aneurysmal recanalization in this series (Table 5).

\section{Discussion}

Despite the proved efficacy of endovascular coil embolization for the treatment of ruptured intracranial aneurysm reported in ISAT, ${ }^{1}$ long-term durability still remains as a major handicap of this treatment technique. The progression of coil devices has occurred in an attempt to maximize occlusion rates and minimize danger. There are 2 main lineages of coil development in response to the perceived market demand: One is mechanical or geometric modification, and the other is chemical modification. Mechanical modification includes stretchresistant coils developed for the prevention of coil stretch or fracture, and complex or 3D coils for the conformational coil 

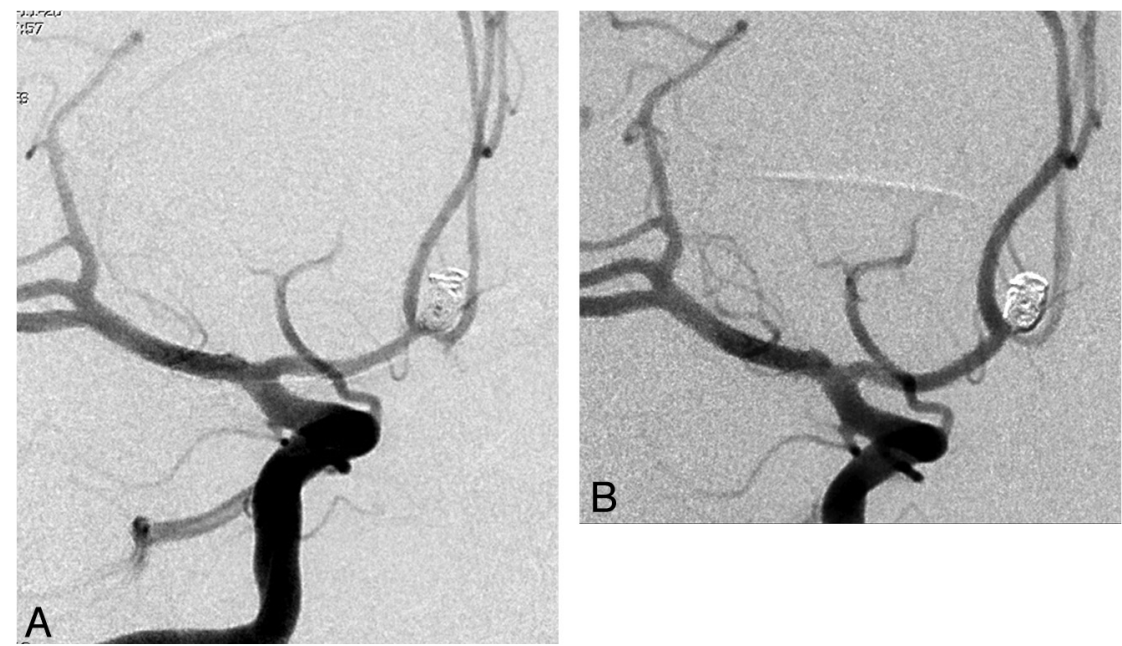

Fig 3. Improved degree of occlusion. A, A 40-year-old man with a ruptured anterior communicating artery aneurysm. Immediate postembolization angiogram demonstrates residual filling into the aneurysmal sac. $B$, An 8-month follow-up angiogram shows complete occlusion of aneurysmal sac.
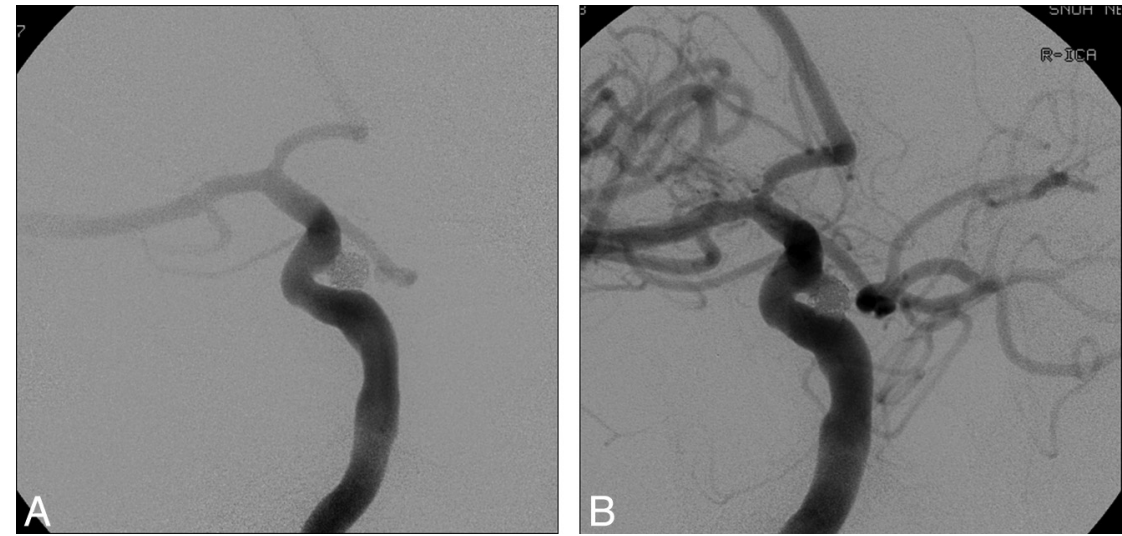

Fig 4. Worsened degree of occlusion. A, A 60-year-old woman with a paraclinoid aneurysm. Immediate postembolization angiogram shows complete occlusion of the aneurysm. $B$, A 1 -year follow-up angiogram reveals minor recanalization at the neck.

Table 4: Cases showing recanalization in HydroSoft coil group

\begin{tabular}{lccccrrr}
\hline $\begin{array}{l}\text { Patient No./Sex/ } \\
\text { Age (yr) }\end{array}$ & $\begin{array}{c}\text { Type of } \\
\text { Recanalization }\end{array}$ & H-H & Location & Size (mm) & PD (\%) & \%HS & Interval (mo) \\
\hline $8 / \mathrm{M} / 47$ & Minor & 1 & SCA & $6.1 \times 5.3 \times 4.9$ & 24 & 64 & 8 \\
$21 / \mathrm{M} / 46$ & Major & 4 & ACA & $3.8 \times 2.4 \times 2.3$ & 73 & 100 & 6 \\
$75 / \mathrm{F} / 63$ & Major & 2 & Basilar & $7.0 \times 7.5 \times 8.5$ & 30 & 51 & 10 \\
\hline
\end{tabular}

$\mathrm{H}-\mathrm{H}$ indicates Hunt and Hess grade; PD, packing density; \% HS, \% length of HydroSoft coils among total coils deployed; Interval, interval from coil embolization to diagnosis of aneurysm recanalization; SCA, superior cerebellar artery; ACA, anterior cerebral artery.

packing of difficult aneurysmal geometries. Chemical modifications or coated coils include PGA or PGLA and hydrogelcoated coils. Some disappointing results, however, have been reported from the series with Matrix detachable coils (Boston Scientific), revealing no different or even higher recanalization rates than those reported for bare platinum coils. ${ }^{1,13}$ HydroCoil results seemed to be promising, showing higher packing attenuation, less coil compaction, and thicker neointimal tissue at the neck of the aneurysm. ${ }^{14}$ However, this hydrogelcoated coil has been reported to be related to aseptic meningitis $^{15}$ and delayed hydrocephalus. ${ }^{3,4}$

The HydroCoil also has some technical limitations. Because of its relative stiffness secondary to hydrogel coating and the time restriction in its deployment, the HydroCoil was considered as a filling coil but not as a finishing coil. ${ }^{16}$ To over-

\begin{tabular}{|c|c|c|}
\hline \multirow[b]{2}{*}{ Variable } & \multicolumn{2}{|c|}{$P$ Value } \\
\hline & $\begin{array}{c}\text { Simple Logistic } \\
\text { Regression } \\
\text { Analysis }\end{array}$ & $\begin{array}{c}\text { Multiple Logistic } \\
\text { Regression } \\
\text { Analysis }\end{array}$ \\
\hline \% length of HydroSoft coils & 0.581 & 0.622 \\
\hline Presentation, ruptured versus unruptured & $0.001^{*}$ & $0.002^{*}$ \\
\hline Maximal aneurysm diameter & 0.890 & 0.440 \\
\hline Aneurysm volume & 0.081 & 0.062 \\
\hline $\begin{array}{l}\text { Aneurysm location, anterior versus } \\
\text { posterior circulation }\end{array}$ & 0.440 & 0.542 \\
\hline $\begin{array}{l}\text { Degree of occlusion, incomplete versus } \\
\text { complete or near complete }\end{array}$ & 0.061 & 0.155 \\
\hline Packing attenuation & 0.417 & 0.103 \\
\hline
\end{tabular}

* Statistically significant 


\begin{tabular}{|c|c|c|c|c|c|}
\hline Series (ref. no.) & $\begin{array}{l}\text { Coating } \\
\text { Material }\end{array}$ & $\begin{array}{c}\text { No. of } \\
\text { Aneurysms }\end{array}$ & $\begin{array}{c}\text { Major } \\
\text { Recanalization }\end{array}$ & $\begin{array}{c}\text { Mean Follow-Up } \\
\text { Interval }\end{array}$ & Study Design \\
\hline Sluzewski et al. ${ }^{20}$ & None & 145 & $28 \%$ & 6 months & Retrospective, single-center \\
\hline Kagn et al. ${ }^{2}$ & PGLA & 39 & $13 \%$ & 6 months & Retrospective, single-center \\
\hline Niimi et al. ${ }^{21}$ & PGLA & 47 & $21 \%$ & 12 months & Retrospective, single-center \\
\hline Gunnarsson et al. ${ }^{22}$ & Hydrogel & 121 & $22 \%$ & 16 months & Retrospective, single-center \\
\hline Current study & Hydrogel & 127 & $2 \%$ & 11 months & Prospective, multicenter \\
\hline
\end{tabular}

PGLA indicates polyglycolic acid/lactide copolymer

come the handicaps of the HydroCoil as a finishing coil, the new HydroSoft coil was developed. Because the HydroSoft coil contains less hydrogel within the coil gaps, this new coil is softer and swells more slowly than the HydroCoil. These properties of the HydroSoft coil make it possible to retrieve or reposition it without time limitations. It expands and fills up to $40 \%$ more volume than a bare platinum coil. Because of all these characteristics of the HydroSoft coil, the authors determined that it could be used as a finishing coil as well as a filling coil.

In this prospective multicenter observational study, the authors intended to show the safety and efficacy of the HydroSoft coil as well as the follow-up results. As to the safety, procedurerelated complications were $4.7 \%$ (6/127 aneurysms), and immediate procedure-related morbidity and mortality rates were $0.8 \%$ and $0 \%$. Systematic review of endovascular coiling of cerebral aneurysms by using coated coils demonstrated $4.7 \%$ and $2.9 \%$ morbidity-mortality rates in PGLA-coated and hydrogel-coated groups, respectively. ${ }^{17}$ Immediate angiographic results of the HydroSoft coil were complete occlusion in 69\%, residual neck in 19\%, and residual sac in 12\%. The HydroCoil Endovascular Aneurysm Occlusion and Packing Study showed complete immediate occlusion in $47 \%$ and residual neck in $31 \%{ }^{18}$ This high rate of complete angiographic occlusion in the HydroSoft coil may be attributed to the properties of less stiffness and slower volumetric expansion, which let this coil be used as a finishing coil as well as a filling coil. Guo et al ${ }^{19}$ reported that the HydroSoft coil showed higher packing attenuation (a mean of $47 \%$ versus $38 \%$ ) and fewer recurrences ( $2.3 \%$ versus $10 \%$ at 6 -month follow-up) in comparison with HydroCoils.

In this HydroSoft coil series, the overall recurrence rate was $3 \%(3 / 105)$ at the mean interval of an 11-month follow-up. Berenstein et $\mathrm{al}^{4}$ reported a $21 \%$ recurrence rate for 53 aneurysms treated with HydroCoils followed for a mean of 10.3 months. Table 6 summarizes some previous series with follow-up results reported in the literature. ${ }^{14,20-22}$ The major recanalization rate ranged from $7 \%$ to $20 \%$. HydroCoils showed high initial aneurysmal occlusion rates and durability at the time of the follow-up evaluation. However, the high incidence of aseptic meningitis and hydrocephalus provoked concern about the safety of this coil device. ${ }^{3}$ In the present study, there was no incidence of aseptic meningitis or related hydrocephalus. The histologic findings of the HydroSoft coil were found to be similar to those of HydroCoil, demonstrating complete endothelialization and neointima formation at the aneurysmal neck surface. ${ }^{16}$ The main difference between the HydroSoft coil and HydroCoil was the thickness of neointima (ie, the HydroSoft coil resulted in thinner neointima). ${ }^{16}$ The HydroSoft coil contains hydrogel, a cross-linked copolymer of acrylamide and sodium acrylate, but has a lower amount than the HydroCoil.

The mechanism of HydroCoil-associated aseptic meningitis and related hydrocephalus is unknown. The reason for no occurrence of aseptic meningitis in the present study of the HydroSoft coil is not known, either. A presumptive explanation could be drawn from the properties of the HydroSoft coil (which contains less hydrogel than the HydroCoil) that eventually lead to less provocation of a histologic response and less volumetric expansion. Meanwhile, long-term clinical follow-up for the occurrence of aseptic meningitis and related hydrocephalus in patients treated with HydroSoft coils would be necessary.

Our study has a limitation in that it limited the size of aneurysms to $<10 \mathrm{~mm}$ in the longest diameter due to the lack of HydroSoft coils of $>6 \mathrm{~mm}$ in diameter. Therefore, we think that the percentage length of the HydroSoft coils is destined to be lowered too much in the treatment of large aneurysms. In those cases, the influence of the HydroSoft coils on the angiographic and clinical outcome could not be measured appropriately. Due to the presence of the limitation in this study, the impressive results of recurrence rate could be diluted. However, even considering that this study was performed for smallor medium-sized aneurysms ( $<10 \mathrm{~mm}$ in longest diameter), the recurrence rate of $3 \%$ is still overwhelming. Another limitation of this study is a relatively short-term follow-up period. Long-term follow-up data are also under collection, and we hope to report the long-term results in the future. In addition, this study was a registry, not a consecutive series of patients treated, and we limited the enrollment to cases satisfying a rather strict criterion (ie, percentage length of the HydroSoft coils used in a certain aneurysm). Further study on a consecutive series is required to define the safety issues related to the HydroSoft coils more clearly.

Finally, we set different inclusion criteria in regard to the percentage length for practical reasons. Basically the HydroSoft coils are designed for filling rather than framing, which means that we usually needed another kind of coil (or coils) other than the HydroSoft coils for initial framing during the endovascular treatment of an aneurysm. In addition, the largest diameter of the HydroSoft coil is $6 \mathrm{~mm}$ at present. When we treated smaller aneurysms, the initial framing coil occupied a considerable volume and length; thus, it was difficult to achieve the percentage length of HydroSoft coils of $>50 \%$ for them. Meanwhile, larger aneurysms require considerable lengths of framing coils and other kinds of filling coils of a larger diameter, which limit the achievable percentage length of the HydroSoft coils. 


\section{Conclusions}

The overall safety profile of HydroSoft coils appears acceptable. HydroSoft coil embolization seems to be favorable compared with that in large series of bare platinum or coated coils in terms of initial occlusion rates and durability at the time of follow-up. Long-term follow-up studies for the evaluation of the durability of HydroSoft coil embolization and monitoring of the clinical outcomes such as aseptic meningitis are needed.

\section{Appendix}

\section{The following investigators and centers participated in this multicenter study:}

Moon Hee Han, Bae Ju Kwon, Hyun-Seung Kang, Young Dae Cho; Seoul National University Hospital, Seoul, Korea

O-Ki Kwon, Cheolkyu Jung; Seoul National University Bundang Hospital, Bundang, Korea

Pyoung Jeon, Keon Ha Kim; Samsung Medical Center, Seoul, Korea

Do-Sung Yoo, Yoo-Dong Won; Uijeongbu St. Mary's Hospital, Uijeongbu, Korea

Tae Hong Lee; Pusan National University Hospital, Pusan, Korea

Sang Hoon Shin, Soon Chan; Kwon Ulsan University Hospital, Ulsan, Korea

Hong Gee Roh; Konkuk University Hospital, Seoul, Korea

Deok Hee Lee; Asan Medical Center, Seoul, Korea

Seung Hun Sheen; Chuncheon Sacred Heart Hospital, Chuncheon, Korea

Hae Wook Pyun; Inha University Hospital, Incheon, Korea

Disclosures: Jae Hyo Park. Research Support (including provision of equipment or materials): MicroVention, CA Inc; Details: financial support for gathering and processing of data for multicenter trial; Moon Hee Han. Consultant. MicroVention and Codman Neurovascular.

\section{References}

1. Molyneux A, Kerr R, Stratton I, et al. International Subarachnoid Aneurysm Trial (ISAT) of neurological clipping versus endovascular coiling in 2143 patients with ruptured intracranial aneurysms: a randomised trial. Lancet 2002;360:1267-74

2. Kang HS, Han MH, Kwon BJ, et al. Short-term outcome of intracranial aneurysms treated with polyglycolic acid/lactide copolymer-coated coils compared to historical controls treated with bare platinum coils: a single-center experience. AJNR Am J Neuroradiol 2005;26:1921-28

3. Kang HS, Han MH, Lee TH, et al. Embolization of intracranial aneurysms with hydrogel-coated coils: results of a Korean multicenter trial. Neurosurgery 2007;61:51-58

4. Berenstein A, Song JK, Niimi Y, et al. Treatment of cerebral aneurysms with hydrogel-coated platinum coils (HydroCoil): early single-center experience. AJNR Am J Neuroradiol 2006;27:1834-40

5. Raymond J, Guilbert F, Weill A, et al. Long-term angiographic recurrences after selective endovascular treatment of aneurysms with detachable coils. Stroke 2003;34:1398-403

6. Yavuz K, Geyik S, Saatci I, et al. Wingspan stent system in the endovascular treatment of intracranial aneurysms: clinical experience with mid-term follow-up results. J Neurosurg 2008;109:445-53

7. Lylyk P, Ferrario A, Pasbon B, et al. Buenos Aires experiences with the Neuroform self-expanding stent for the treatment of intracranial aneurysms. $\mathrm{J} \mathrm{Neu}$ rosurg 2005;102:235-41

8. Cekirge HS, Saatci I, Ozturk MH, et al. Late angiographic and clinical follow-up results of 100 consecutive aneurysms treated with Onyx reconstruction: largest single-center experience. Neuroradiology 2006; 48:113-26

9. Sluzewski M, van Rooij WL, Beute GN, et al. Balloon-assisted coil embolization of intracranial aneurysms: incidence, complications, and angiography results. J Neurosurg 2006;105:396-99

10. Kwon BJ, Han MH, Oh CW, et al. Anatomical and clinical outcomes after endovascular treatment for unruptured cerebral aneurysms: a single center experience. Intervent Neuroradiol 2002;8:367-76

11. Kwon BJ, Han MH, Oh CW, et al. Procedure-related haemorrhage in embolism of intracranial aneurysms with Guglielmi detachable coils. Neuroradiology 2003;45:562-69

12. Roy D, Milot G, Raymond J. Endovascular treatment of unruptured aneurysms. Stroke 2001;32:1998-2004

13. Fiorella D, Albuquerge FC, McDougall CG. Durability of aneurysm embolization with Matrix detachable coils. Neurosurgery 2006;58:51-59

14. Kallmes DF, Fujiwara NH. New expandable hydrogel-platinum coil hybrid device for aneurysm embolization. AJNR Am J Neuroradiol 2002;23:1580-88

15. Lim SH, Han MH, Kwon BJ, et al. Aseptic meningitis after embolization of cerebral aneurysms using hydrogel-coated coils: report of three cases. AJNR Am J Neuroradiol 2007;28:511-12

16. Tsumoto T, Niimi Y, Berenstein A. Evaluation of the new HydroSoft coil in a canine model of bifurcation aneurysm. J Neurosurg 2009;111:11-16

17. White PM, Raymond J. Endovascular coiling of cerebral aneurysms using "bioactive" or coated-coil technologies: a systematic review of the literature. AJNR Am J Neuroradiol 2009;30:219-26. Epub 2008 Oct 8

18. White PM, Lewis SC, Nahser H, et al. HydroCoil Endovascular Aneurysm Occlusion and Packing Study (HELPS trial): procedural safety and operatorassisted efficacy results. AJNR Am J Neuroradiol 2008;29:217-23

19. Guo XB, Fan YM, Zhang JN. HydroSoft coil versus HydroCoil for endovascular aneurysm occlusion study: a single-center experience. Eur J Radiol 2010;10:1016-20

20. Sluzewski M, van Rooij WJ, Slob MJ, et al. Relation between aneurysm volume, packing, and compaction in $\mathbf{1 4 5}$ cerebral aneurysms treated with coils. Radiology 2004;231:653-58

21. Niimi Y, Song J, Madrid M, et al. Endosaccular treatment of intracranial aneurysms using Matrix coils: early experience and midterm follow-up. Stroke 2006;37:1028-32

22. Gunnarsson T, Tong FC, Klurfan P, et al. Angiographic and clinical outcomes in 200 consecutive patients with cerebral aneurysm treated with hydrogelcoated coils. AJNR Am J Neuroradiol 2009;30:1657-64 